\title{
Diacronie
}

Studi di Storia Contemporanea

$\mathrm{N}^{\circ} 24,4 \mid 2015$

Le dittature militari: fisionomia ed eredità politica

\section{Corone, guerrieri e redentori}

Il culto della personalità nelle dittature militari dell'Africa subsahariana negli anni della Guerra Fredda

\section{Claudio Mancuso}

\section{Q OpenEdition}

1 Journals

\section{Edizione digitale}

URL: http://journals.openedition.org/diacronie/3845

DOI: 10.4000/diacronie.3845

ISSN: 2038-0925

Editore

Association culturelle Diacronie

Notizia bibliografica digitale

Claudio Mancuso, « Corone, guerrieri e redentori », Diacronie [Online], № 24, 4| 2015, documento 17, Messo online il 29 décembre 2015, consultato il 01 mai 2019. URL : http://journals.openedition.org/ diacronie/3845 ; DOI : 10.4000/diacronie.3845 


\section{Diacronie}

\section{$17 /$}

\section{Corone, guerrieri e redentori}

\section{Il culto della personalità nelle dittature militari dell'Africa subsahariana negli anni dellla Guerra Fredda}

Claudio MANCUSO *

Uno degli aspetti più controversi dei processi di decolonizzazione che hanno interessato l'Africa riguarda senz'altro il ruolo dei militari nelle trasformazioni politiche e nella formazione dei nuovi stati indipendenti. All'interno delle dinamiche legate al consolidamento dei governi dittatoriali assume un ruolo cruciale la questione della costruzione del culto della personalità. Quale ruolo ebbe l'esaltazione della figura del leader nella ricerca di una legittimazione per il potere conquistato con le armi? Quali i meccanismi di propaganda messi in atto per promuovere tale culto? Il contributo propone lo studio in chiave comparativa di questi fenomeni relativamente alle diverse dittature militari instauratesi nell'area subsahariana del continente africano nei decenni della guerra fredda.

Il potere non è un mezzo, è un fine. Non si stabilisce una dittatura nell'intento di salvaguardare una rivoluzione; ma si fa una rivoluzione nell'intento di stabilire una dittatura. Il fine della persecuzione è la persecuzione. Il fine della tortura è la tortura. Il fine del potere è il potere. (George Orwell, 1984) e complesse dinamiche relative ai processi di decolonizzazione in Africa inserite spesso all'interno di cornici geopolitiche più ampie ${ }^{1}$ - hanno determinato in molti casi il collasso delle nuove compagini indipendenti e

${ }^{1}$ Cfr. ARNOLD, Guy, Historical Dictionary of Civil Wars in Africa. Second Edition, Lanham, The Scarecrow Press, 2008, p. X. 
l'avvento di regimi dittatoriali a carattere militare ${ }^{2}$. A partire dalla metà degli anni Sessanta - soprattutto nella fascia subsahariana - una lunga serie di colpi di stato ha posto gli eserciti al centro della vita politica del continente, incidendo a fondo sui processi di democratizzazione intrapresi dai paesi africani3.

A lungo impegnata nell'analisi dei fattori che hanno favorito l'irruzione dei pretoriani sulla scena politica, la ricerca storiografica, specialmente a partire dagli ultimi decenni, ha spostato il campo d'indagine verso le fasi successive all'avvento di tali regimi, portando in primo piano le questioni legate alle strategie di consolidamento e di legittimazione dei nuovi sistemi autoritari ${ }^{4}$. In questo quadro gli studiosi hanno attribuito una crescente attenzione al ruolo della dimensione sociale e simbolica, consapevoli del fatto che tali processi occupano una posizione tutt'altro che marginale non soltanto nella definizione dei contesti politici, ma anche nell'analisi delle trasformazioni culturalis.

Proprio in questa prospettiva si colloca un aspetto spesso poco indagato, ovvero la questione del culto della personalità,

the glorification and sometimes near-deification of the great national leader [...]. In schoolbooks, in the press, and in the official propaganda of government and party [...] they became the particular objects of hero-worship. They were hailed as the fathers of their nations; considered wise in the ways of understanding the best interests of their peoples; and [...] endowed with an almost supernatural aura ${ }^{6}$.

Le principali difficoltà che hanno ostacolato la trattazione di queste tematiche riguardano da un lato la scarsità di fonti o l'impossibilità ad accedervi, dall'altro lato le numerose mistificazioni coagulatesi intorno a queste figure controverse, dovute spesso

${ }^{2}$ Su questi temi si veda MAZRUI, Ali A., «The Blood of Experience: The Failed State and Political Collapse in Africa», in World Policy Journal, 12, 1/1995, pp. 28-34.

3 Numerosi i lavori sul ruolo dei militari nell'Africa postcoloniale, tra gli altri si vedano BAYNHAM, Simon (ed.), Military Power and Politics in Black Africa, New York, St. Martin's Press, 1986; BIENEN, Henry, Armed Forces, Conflict and Change in Africa, Boulder, Westview Press, 1989; DECALO, Samuel, Coups and Army Rule in Africa: Motivations and Constraints, New Haven, Yale University Press, 1990. In chiave comparativa, con riferimenti anche al peso della dimensione militare nelle società africane tradizionali, cfr. ONWUMECHILI, Chuka, African Democratization and Military Coups, Westport, Praeger, 1998.

4 Cfr. PASQUINO, Gianfranco, Introduzione, in NORDLINGER, Eric A., I nuovi pretoriani, Milano, ETAS, 1978, pp. 4-5.

5 Cfr. FOLEY, Michael, Political Leadership: Themes, Contexts and Critiques, Oxford, Oxford University Press, 2013, p. 3. Per quanto riguarda il contesto africano, un'interessante riflessione sull'importanza del mito e del rituale nella costruzione del potere nelle società postcoloniali si trova in COMAROFF, Jean, COMAROFF, John. L. (eds.), Modernity and Its Malcontents: Ritual and Power in Postcolonial Africa, Chicago, Chicago University Press, 1993.

${ }^{6}$ LE VINE, Victor T., Politics in Francophone Africa, Boulder, Lynne Rienner, 2004, pp. 105106. 
al fatto che «il comportamento dei capi di stato dell'Africa indipendente [...] esercita un insolito livello di fascinazione su una non meno insolitamente diffusa gamma di lettori. Le loro caratteristiche e le loro ideologie, i loro eccessi, le loro eccentricità [...], il loro modo esagerato di vivere e la loro maniera spesso brutale di morire»7, hanno fornito numerosi spunti di analisi.

Sebbene una lunga tradizione di studi abbia attribuito un'importanza ristretta al culto e al carisma della leadership in quanto fattore di legittimazione dei governi pretoriani, occorre evidenziare come la ritualità legata alla figura del dittatorepresidente costituisca uno dei luoghi ideali d'incontro tra il popolo e il potere, soprattutto in un contesto culturale come quello dell'Africa subsahariana ${ }^{8}$. All'interno di un sistema politico fondato sulla militarizzazione della società e, in molti casi, sulla prassi del terrore e della violenza, i meccanismi di rappresentazione del potere e, quindi, l'ostentazione della figura del leader rappresentano dei canali comunicativi fondamentali e degli strumenti di legittimazione indispensabili9.

Nondimeno, la crucialità di questa tematica è testimoniata anche dalla sua diretta connessione con altre due questioni fondamentali nel quadro dello sviluppo storicopolitico dell'Africa contemporanea. In primo luogo, il problema del consolidamento dell'unità nazionale: in un continente in cui «quasi ogni stato appare fragile a causa della mancanza di un'identità nazionale o di un senso del patriottismo in grado di tenere insieme il paese ${ }^{10}$, il culto della personalità rappresenta non soltanto uno strumento di creazione del consenso e di giustificazione del potere conquistato, ma anche un punto di riferimento imprescindibile nella costruzione di un'identità nazionale condivisa. In seconda istanza, la questione delle trasformazioni imposte dai processi di militarizzazione alla leadership politica nel continente africano ${ }^{11}$ : il modello del capo militare che ha conquistato il potere affermandosi come dittatore finisce per sovrapporsi e sostituirsi all'icona del politico rivoluzionario che aveva guidato il suo

7 KIRK-GREENE, Anthony H. M., «His eternity, His eccentricity, or His exemplarity? A Further Contribution to the Study of H. E. the African Head of State», in African Affairs, 90, 1991, pp.163-187, p. 163.

8 Lo stesso Eric Nordlinger, fautore di un rinnovamento nell'analisi del fenomeno del pretorianesimo, dimostra un certo scetticismo nella considerazione di questi fattori, affermando che «le forze armate sono tutt'altro che terreno favorevole alla produzione di carisma a causa delle loro caratteristiche burocratiche e delle regole di anzianità». NORDLINGER, Eric A., I nuovi pretoriani, cit., p. 168.

9 Cfr. NWANKWO, Arthur Agwuncha, African Dictators: the Logic of Tyranny and Lessons from History, Enugu, Fourth Dimension, 1998.

${ }_{10}$ RUBIN, Barry, Modern Dictators: Third World Coup Makers, Strongmen, and Populist Tyrants, New York, McGraw-Hill, 1987, p. 87.

${ }_{11}$ Cfr. CARTWRIGHT, John, Political Leadership in Africa, London, Croom Helm, 1983; VAN WYK, Jo-Ansie, «Political Leaders in Africa. Presidents, Patrons or Profiteers?», in Occasional Paper Series, 2, 1/2007, pp. 3-38. 
popolo all'indipendenza dal giogo europeo. L'immagine del padre della patria è così soppiantata da quella del padrone dello stato.

I contributi legati a questo argomento appaiono dunque ancora esigui, soprattutto in chiave comparativa, anche a causa delle caratteristiche assai diverse e frammentarie assunte dai governi militari, determinate non soltanto dalle dinamiche imposte dalle ideologie politiche, ma ancor più spesso dalla dimensione personale assunta dalla leadership ${ }^{12}$.

Il presente articolo, pertanto, ha come obiettivo quello di stimolare il dibattito scientifico su questi aspetti, provando a individuare all'interno di un cluster così ampio di variabili, delle caratteristiche comuni nella costruzione di un culto del capo.

\section{Il potere dei nomi e la rappresentazione della leadership}

Le strategie politiche e comunicative sottese alla creazione di un culto della leadership pongono in primo piano la questione del rapporto con l'opinione pubblica. Le dittature militari oggetto della mia analisi - sebbene con esiti differenti attribuiscono a questa esigenza un'importanza cruciale, e uno dei primi aspetti che emerge riguarda le modalità di rappresentazione della figura del despota attraverso la manipolazione linguistica e semantica dei nomi e dei titoli. Il nome infatti è lo strumento comunicativo più immediato e quindi deve contenere in sé i caratteri fondamentali che il leader vuole conferire alla propria persona ${ }^{13}$.

L'esempio più eclatante sotto questo profilo riguarda il dittatore congolose Mobutu, il quale cambiò il proprio nome da Joseph-Désiré Mobutu a Mobutu Sese Seko Koko Ngbendu Wa Za Banga, che nella lingua locale significa "Il guerriero onnipotente che non conosce sconfitta a causa della sua resistenza e della sua inflessibile volontà, e che lascia il fuoco nella sua scia mentre va di vittoria in vittoria"14. L'assunzione di un nuovo

12 Cfr. DECALO, Samuel, Psychoses of Power. African Personal Dictatorships, Boulder, Westview Press, 1989; EZROW, Natasha M., FRANTZ, Erica, Dictators and Dictatorships: Understanding Authoritarian Regimes and their Leaders, New York, Continuum, 2011; JACKSON, Robert H., ROSBERG, Carl J., Personal Rule in Black Africa: prince, autocrat, prophet, tyrant, Berkeley-Los Angeles, California University Press, 1982.

${ }^{13}$ Questa attenzione per i titoli attribuiti alla leadership politica appare diffusa anche in altri sistemi politici africani. Ad esempio, nello Swaziland il sovrano veniva definito ngwenyama, ovvero "Il Leone". In Etiopia l'imperatore era chiamato Seyum Egziabher, che significa "L'Eletto di Dio". Infine, Kwame Nkrumah, leader politico ghanese, regolarmente eletto, si autodefinì come Osagyefo, cioè "Il Redentore". Cfr. VAN WYK, Jo-Ansie, "Political Leaders in Africa. Presidents, Patrons or Profiteers?», cit., p. 11.

${ }_{14}$ Sulla traduzione del nome si veda CARBONE, Giovanni, L'Africa. Gli stati, la politica, $i$ confini, Bologna, Il Mulino, 2005, p. 115; ELLIOT, Jeffrey M., DYMALLY Mervyn M. (eds.), Voices of Zaire: Rhetoric or Reality, Washington, Washington Institute Press, 1990. 
nome rappresenta un passaggio fondamentale nella venerazione della persona del presidente, in quanto ne sancisce la legittimazione semantica, tramite il ricorso a una terminologia legata alla cultura congolese ancestrale e per questo ancora più potente $\mathrm{e}$ profonda ${ }^{15}$.

Oltre al proprio nome, il dittatore cambiò anche quello della sua nazione, e, in luogo del toponimo coloniale Congo, che rimandava alla dominazione belga, introdusse a partire dal 1971 il nome Zaire (la bandiera del nuovo stato fu altresì sostituita con quella del partito del presidente, il "Mouvement Populaire de la Revolution") ${ }^{16}$. L'impulso ridenominativo fu esteso all'intera geografia del paese, promuovendo la riscoperta degli antichi nomi etnici. Così, ad esempio, mutarono i toponimi che designavano le principali città del Paese: la capitale Leopoldville assunse il nome di Kinshasa, Stanleyville quello di Kisangani, mentre Elisabethville fu trasformato in Lubumbashi, e perfino il nome del lago Alberto fu ribattezzato in onore del dittatore e divenne lago Mobutu Sese Seko. Come evidenzia Eric Nordlinger, «questa commistione di astuzia politica, rappresentazione pubblica e impegno [...] per la risurrezione delle antiche tradizioni del paese ha grandemente contribuito a consolidare l'autorità del presidente e a legittimare il suo governo» ${ }^{17}$.

Nondimeno, a partire dal 1973, dopo un viaggio in Cina e in Corea del Nord, Mobutu assunse i titoli di "Timoniere Illuminato" e di "Padre della Nazione", a sottolineare ancora una volta le ragioni che stavano alla base del culto che ogni zairese doveva tributargli ${ }^{18}$. Nel corso dei decenni altri appellativi quali "Il Messia", "Il Redentore", "Il Salvatore delle genti" lo collocarono in una dimensione sempre più sacra $^{19}$. La sua attenzione per il nome e la parola raggiunse livelli tali che alla stampa

\footnotetext{
${ }_{15}$ Come sottolinea Victor T. Le Vin, «one common phenomenon throughout francophone Africa - as in most other African states - has been the attempt to provide national myths of historical origin and thereby quasitraditional legitimacy for the new polities [...]. The reconstructed emphasis is [...] on the glories of past kingdoms and empires (Macina, Mali, Ghana, Songhai, etc.); the rich traditional past; and probably most important, the heroic role of such colonial-era resistance leaders». LE VINE, Victor T., Politics in Francophone Africa, cit., p. 105. Su questi aspetti cfr. anche NORDLINGER, Eric A., I nuovi pretoriani, cit., pp. 168-171.

${ }^{16}$ Cfr. HESSELBEIN, Gabi, «The Rise and Decline of the Congolese State: an analytical narrative on state-making», in Working Paper, 21, 2/2007, p. 27.

${ }_{17}$ NORDLINGER, Eric A., I nuovi pretoriani, cit., p. 171. Si veda anche DUBOIS, Victor, «Zaire under President Sese Seko Mobutu: The Return to Authenticity», in American Universities Fieldstaff Reports. Central and Southern Africa Series, 17, 1/1973.

${ }^{18}$ Cfr. RUBIN, Barry, Modern Dictators, cit., p. 94.

19 Cfr. The Independent, 9 settembre 1997, URL:

< http://www.independent.co.uk/news/people/obituary-mobutu-sese-seko-1238238.html > [consultato il 27 agosto 2015].
} 
ufficiale dello Zaire fu vietato, a partire dal 1975, di menzionare il nome di qualsiasi individuo che non fosse Mobutu stesso ${ }^{20}$.

Fortemente connotati in chiave sacrale anche gli appellativi di Ali Soilih, leader delle Comore, che si faceva chiamare "Dio"; di Étienne Eyadéma Gnassingbé, dittatore del Togo, che si presentava come l'“Eletto di Dio”"21; e, soprattutto, di Francisco Maciàs Nguema, despota della Guinea Equatoriale, il quale assunse una lunghissima serie di titoli, tra i quali "Pietra angolare del dogma unico della nostra nazione", "Unico miracolo nazionale", "Gran maestro dell'educazione, scienza e cultura", "Leader d'acciaio", "Padre della nostra indipendenza e libertà", "Architetto supremo della Guinea Equatoriale", "Supremo inquisitore del colonialismo", "Leader invincibile" e "Gran messia"; nondimeno, nel 1978 cambiò il motto nazionale in "Non c'è altro Dio che Maciàs Nguema”22. Anche colui che defenestrò Maciàs Nguema, il nipote Teodoro Obiang Nguema Mbasogo - tuttora al potere -, proseguì sulla strada di un autoritarismo sacrale, proclamandosi dio e attribuendosi vari titoli che si inseriscono sempre all'interno di questa dimensione magico-esoterica ${ }^{23}$.

Sul fronte della metamorfosi nomenclativa agì anche il dittatore ugandese Idi Amin Dada, il quale mise in scena un'ampia galleria di appellativi con i quali si presentava al suo popolo. Tra questi "Big Daddy", a simboleggiare il fatto di essere come un padre amorevole per l'Uganda, ma anche titoli molto più eccentrici, come "Sua Eccellenza, Presidente a vita, Feldmaresciallo Al Hadji Dottor Idi Amin Dada, Croce Vittoriosa, Ordine del Servizio Distinto, Croce Militare, Signore di tutte le bestie della terra e dei pesci del mare e Conquistatore dell'Impero Britannico in Africa in generale e in Uganda in particolare", o quello di "Re di Scozia"24. In questo caso, sul piano linguistico, il tiranno manifestava il duplice obiettivo di apparire come un signore onnipotente e di evidenziare la cessata sudditanza con l'ex madrepatria inglese.

Jean-Bedel Bokassa, dittatore della Repubblica Centrafricana, espresse nei vari titoli attribuitisi tutta la propria sete di potere, passando rispettivamente da colonnello

${ }^{20}$ Cfr. EDGERTON, Robert, The Troubled Heart of Africa: A History of the Congo, New York, St. Martin's Press, 2002; YOUNG, Crawfor, TURNER, Thomas, The Rise and Decline of the Zairian State, Madison, Wisconsin University Press, 1985, p. 169.

${ }^{21}$ Cfr. DECALO, Samuel, Psychoses of Power. African Personal Dictatorships, cit.

${ }^{22}$ Cfr. BUALE BORIKÓ, Emiliano, El laberinto guineano, Madrid, Iepala, 1989, p. 103.

${ }_{23}$ Cfr. Ibidem, pp. 104-107.

${ }^{24}$ Cfr. KYEMBA, Henry, A State of Blood: The Inside Story of Idi Amin, New York, Paddington, 1977; MOGHAL, Manzoor, Idi Amin. Lion of Africa, Bloomington, AuthorHouse, 2010; WOODWARD, Peter, "Ambiguous Amin", in African Affairs. Journal of The Royal African Society, 77, 307, 1978, pp. 153-164. 
a generale, quindi maresciallo, poi presidente a vita e infine imperatore, col nome di "Imperatore del Centrafrica per volontà del popolo Centrafricano"25.

Singolare invece l'appellativo con il quale Ahmed Mathieu Kérékou, dittatore del Benin, si autodefiniva, ovvero il "Camaleonte", accompagnando questa definizione al motto "Il ramo non si spezza tra le zampe del camaleonte". In questo caso il riferimento a una simbologia legata al mondo animale non presuppone il richiamo a una condizione di forza fisica o di possanza, ma intelligenza e duttilità politica, sottolineando il fatto che soltanto le sua capacità avrebbero impedito al fragile equilibrio del Benin di rompersi ${ }^{26}$.

Il dittatore somalo Mohammed Siad Barre era invece definito il "Vecchio". Con questo termine tuttavia non si faceva riferimento all'età del leader, ma si trattava di un'espressione affettiva che designa colui che unisce nell'amore e che guida il popolo verso la saggezza e la giustizia ${ }^{27}$.

France-Albert René, longevo dittatore delle Seychelles si faceva chiamare invece "The Boss" 28 , mentre Juvénal Habyarimana, dittatore ruandese, era soprannominato "Kinani", una parola che nella lingua locale significa "invincibile". Nondimeno, la propaganda favorì la costruzione di un’interessante etimologia del suo nome,

which derives from the verb kubyara (to engender) and the noun imaana, which together could be translated as: "It is God who gives life". Nothing could have been more appropriate in a Catholic, anti-abortion, and basically pro-natalist country, yet very few names could resonated so well with the more traditional themes of fertility, prosperity, and good luck, the "diffuse fecundating fluid" 29.

Molto più legati - sul piano linguistico - alle radici pretoriane i dittatori della Liberia, Samuel Kanyon Doe, del Niger, Seyni Kountché, e, soprattutto, del Burundi, Michel Micombero, il quale veniva chiamato "S. E. Il Colonnello" o "S. E. Il Luogotenente Generale”. Inoltre, sui ritratti ufficiali esposti obbligatoriamente in tutti

25 Cfr. PÉAN, Pierre, Bokassa 1er, Paris, A. Moreau, 1977, pp. 35-40.

${ }_{26}$ Cfr. BANÉGAS, Richard, La démocratie à pas de camélèon: transition et imaginaires politiques au Bénin, Paris, Karthala, 2003; CLAFFEY, Patrick, «Kérékou the Chameleon, Master of Myth», in STRAUSS, Julia C., O'BRIEN, Donal Cruise (eds.), Staging Politics. Power and Performance in Asia and Africa, London, Tauris, 2007, pp. 91-110.

27 Cfr. PESTALOZZA, Luigi, Somalia, cronaca di una rivoluzione, Bari, Dedalo, 1973. Di una delle interviste realizzate dal giornalista italiano al dittatore somalo esiste anche un video alla URL: < https://www.youtube.com/watch?v=UiAIswl9AvQ > [consultato il 4 settembre 2015].

28 Cfr. BOLLÉE, Annegrette, "Language policy in the Seychelles and its consequences», in International Journal of the Sociology of Language, 102, 1993, pp. 85-100.

${ }_{29}$ TAYLOR, Christopher C., «Kings or Presidents? War and the State in Pre- and Post-Genocidal Rwanda», in KAPFERER, Bruce (ed.), State, Sovereignity, War. Civil Violence in Emerging Global Realities, Oxford, Berghahn Books, 2004, p. 130. 
gli edifici e gli esercizi pubblici comparivano i titoli di "Primo presidente della Repubblica del Burundi, liberatore del popolo Murundi"3o. In questo caso, dunque, attraverso il nome e i titoli, il dittatore voleva esaltare proprio la dimensione militare del culto a lui tributato, stabilendo un legame diretto tra il colpo di stato e la liberazione del popolo.

\title{
2. Le radici del culto: propaganda e legittimazione
}

Se la venerazione della persona del leader rappresenta uno snodo fondamentale nella legittimazione del potere conquistato, la maggior parte dei dittatori manifesta un'altrettanto forte attenzione verso la ricerca e l'elaborazione di fattori in grado di motivare e giustificare tale culto agli occhi dell'opinione pubblica. I percorsi intrapresi dai vari capi militari in questa direzione risultano molteplici e, in alcuni casi, contraddittori.

Durante un consiglio dei ministri del 1974, il dittatore ugandese Idi Amin Dada metteva in cima all'agenda politica del suo governo proprio la questione del culto dovuto alla sua persona, attribuendovi una finalità - e quindi una legittimazione - di tipo pedagogico:

\begin{abstract}
As a minister, governor, high-ranking peoples, and the people of the country, they must love their leader. This is the point number one. If you go to any country, you will find that everybody must love his leader. They must love their ministers. They must love their governors. They must love their district commissioners, their teachers, their chiefs. Everybody must be loved. This... It is an education to the people. This is the duty of the minister. If I see the minister is coward, automatically I kick you out of my office, because I know you have got something wrong with you [...]. You must teach people to love their leader, this is the only important, like any country ${ }^{31}$.
\end{abstract}

L’insistenza, a tratti esasperata, sull'idea del rispetto, dell'amore reso al presidente mostrava un avallo politico, in quanto passaggio cruciale nell'educazione del popolo e nella sua crescita morale. Nondimeno, la glorificazione del generale Amin era

30 Cfr. CHRÉTIEN, Jean-Pierre, DUPAQUIER, Jean-François, Burundi 1972. Au bord des génocides, Paris, Khartala, 2007, p. 49.

${ }^{31}$ SCHROEDER, Barbet, Général Idi Amin Dada. Autoportrait, Figaro Films, France, 1974, 93'. 
giustificata dalla sua identificazione con la nazione stessa, con l'Uganda ${ }^{32}$. Adorare e amare il presidente significava adorare e amare il proprio paese. Questa assimilazione appariva come necessaria, e, soprattutto, come manifestazione della volontà popolare:

It is not my intention to be a president, but it is people who appointed me to be the president of Republic of Uganda. The same people have requested me to be the life president of Uganda for what I have done for the country, economically, and all the developments which are now taking place in Uganda. But not my intention33.

Su una prospettiva paternalistica fa leva Robert Mugabe, un'altra figura chiave del periodo della decolonizzazione africana ${ }^{34}$. L'attuale dittatore dello Zimbabwe ha messo in scena a partire dalle prime elezioni che avevano sancito l'indipendenza dello stato, una crescente e progressiva concentrazione di poteri nella propria persona. Dopo essersi proclamato al di sopra della legge35, esplicitò la sua filosofia di governo risolvendo i concetti di partito unico e di nazione nella sua persona. In un discorso del luglio 1985 difatti affermava: «We are one family, one country with one nation, one government. And so we must have one party. It's that simple.... We believe in the inexorable law of unity. You must be united or else you can be divided and perish»36. La rivendicazione di un modello di impronta familistica per l'amministrazione dello Zimbabwe legittima la concentrazione di potere nelle sue mani e il culto a lui tributato proprio in quanto capo della nazione-famiglia, padre indiscusso della patria. Il rapporto che si realizza tra Mugabe e i cittadini zimbabwiani è dunque assimilabile a quello che si instaura tra un padre e i suoi figli; un rapporto in cui lo stato e le istituzioni si trasformano in un possedimento personale del leader 37.

Un'altra dimensione sulla quale hanno fatto leva i dittatori per legittimare la costruzione di un culto della personalità è quella etica. Ł̀, ad esempio, il caso del dittatore ghanese Jerry Rawlings, il quale presentava la propria persona come la sola in

$3^{2}$ Tale identificazione è ben sottolineata in MACDONALD, Kevin, The Last King of Scotland, 2oth Century Fox, United Kingdom, 2006, 121'.

33 SNOW, Jon, Reflections. Idi Amin, 1974, URL: < https://www.youtube.com/watch?v=2vFyYggn1EU > [consultato il 15 luglio 2015].

34 Sebbene non si tratti di una dittatura originata da un colpo di stato dell'esercito, tuttavia la componente militare assume un ruolo chiave nel regime di Mugabe, il quale del resto era stato a capo dell'ala paramilitare dello Zimbabwe African National Union. Cfr. MEREDITH, Martin, Mugabe: Power, Plunder and the Struggle for Zimbabwe's Future, New York, Public Affairs, 2007.

35 Cfr. ARNOLD, James R., WIENER, Roberta, Robert's Mugabe Zimbabwe, Minneapolis, Twenty-First Century Books, 2008, p. 58.

${ }^{36}$ RUBIN, Barry, Modern Dictators, op. cit., p. 116.

37 Cfr. SCHATZBERG, Michael G., Political Legitimacy in Middle Africa: Father, Family, Food, Bloomington, Indiana University Press, 2001, p. 1. 
grado di combattere la corruzione dei precedenti governi, ergendosi a modello di integrità morale e per questo degno di venerazione ${ }^{38}$.

Un potente fattore di legittimazione a cui pretoriani hanno attinto per consolidare il culto legato alla propria immagine è poi quello religioso. La dimensione legata al sacro assume infatti un valore cruciale in un contesto culturale come quello africano, in cui le relazioni religiose costituiscono il principale strumento di interazione sociale39. È questo il modello seguito dal dittatore zairese Mobutu. Il concetto è ben espresso dal suo ministro degli interni Engulu Baanga Mpongo, il quale affermò durante una manifestazione politica:

Dieu a envoyé un grand prophète, notre Mobutu prestigieux Guide. Ce prophète est notre libérateur, notre Messie. Notre Église est le MPR. Son chef est Mobutu. Nous le respectons comme on respecte un pape. Notre évangile est mobutisme. C'est pourquoi le crucifix doit être remplacé par l'image de notre messie ${ }^{40}$.

Dio aveva scelto Mobutu per guidare lo Zaire, ma i poteri del dittatore andavano ben oltre quelli di un leader profetico: non solo è proposta una lettura in chiave cristiana del culto della personalità ma, addirittura, è auspicata una vera e propria sostituzione della religione cristiana con la fede nella persona del presidente. Il leader congolese, in un rapporto di competizione culturale e simbolica con le chiese cristiane (in particolare con quella cattolica), assunse e risolse nella sua persona gli attributi della sacralità, in modo da escludere qualsiasi altra forma di venerazione diversa da quella a lui riservata. Questo cruciale passaggio ideologico fu sancito dall'approvazione della carta costituzionale del 1974, che stabiliva la totale ed esclusiva supremazia del partito di Mobutu, unica istituzione esistente nel paese, incarnata nella persona del presidente $^{41}$.

Fece leva sulla dimensione magico-religiosa anche il dittatore equatoguineano Nguema. Indicativo, da questo punto di vista, il fatto che, nel 1979, in occasione del suo arresto e della sua condanna a morte, nessun soldato dell'esercito volle partecipare alla

${ }^{8}$ Cfr. NUGENT, Paul, Big Men, Small Boys and Politics in Ghana: Power, Ideology and the Burden of History, 1982-1994, London-New York, Pinter, 1995.

39 Cfr. ELLIS, Stephen, TER HAAR, Gerrie, Worlds of Power: Religious Thought and Political Practice in Africa, New York, Oxford University Press, 2004, p. 2.

4o ODENY, Manuel, The Great Plunderer: Mobutu Desire Sese Seko, Zaire, 19 gennaio 2010. URL: < http://theburningsplint.blogspot.it/2010/o1/great-plunderer-mobutu-desire-seseseko.html > [consultato il 7 agosto 2015].

${ }^{41}$ Cfr. YOUNG, Crawfor, TURNER, Thomas, The Rise and Decline of the Zairian State, cit., p. 70 . 
sua esecuzione capitale, in quanto era diffusa la credenza nei presunti poteri magici posseduti dal presidente, il quale fu così giustiziato da truppe marocchine ${ }^{42}$.

Alla dimensione esoterico-soprannaturale fece riferimento anche Eyadéma. Il dittatore togolese sfruttò, da questo punto di vista, un fallito attentato aereo avvenuto il 24 gennaio 1974, trasformando un chiaro sintomo della fragilità del suo potere in un'abile operazione di propaganda politica e di promozione del culto della personalità. Egli infatti decretò la commemorazione annuale di quella data attraverso l'istituzione della "festa della vittoria sulle forze del male", e affermò - falsamente - di essere stato l'unico sopravvissuto ${ }^{43}$. Nella leggenda costruita dal dittatore, i piani imperialisti degli avversari non erano riusciti a sopraffarlo grazie ai suoi poteri soprannaturali, alla sua forza sovraumana e alla sua tempra da eroe. Eyadéma riuscì attraverso la manipolazione in chiave mitologica della realtà storica a trovare una legittimazione soprannaturale per la sua leadership44.

È, tuttavia, il caso centrafricano ad apparire, da questa prospettiva, tra i più controversi e problematici. Infatti, i canali utilizzati da Bokassa nel tentativo di costruire una base di consenso e di sostegno alla propria leadership politica furono molteplici, anche se messi in campo in maniera spesso contraddittoria. Innanzitutto fece riferimento al sacro come strumento di propaganda:

les thémes "chrétiens" de la propagande de Terre Africaine, où l'on compare Bokassa a Boganda, premier prêtre centrafricain, ou même a Jésus-Christ, ne manquent pas. E. Fatrane écrit dès 1966: «Le cœur de Bokassa saigne comme le Christ avant la multiplication des pains et des poissons. Il dit en lui-même, j'ai pitié de la foule, de mes enfants privés de viande»45.

La relazione simbolica istituita con la dimensione evangelica gli permetteva di presentarsi agli occhi dei cittadini come un padre, centrando quindi l'intero apparato

42 Cfr. SUNDIATA, Ibrahim K., Equatorial Guinea. Colonialism, State Terror, and the Search for Stability, Boulder, Westview Press, 1990.

43 Sul luogo del disastro, nei pressi di Sarakawa, fu perfino eretto un monumento (dono della Corea del Nord) che rappresenta il dittatore togolese. Ai piedi della statua i ritratti dei militari caduti durante l'attentato.

${ }_{44}$ Cfr. PACKER, George, The Village of Waiting, New York, Farrar, Straus and Giroux, 1984; RAMSAY, Jeffress F., Africa. Global Studies Series. Seventh Edition, New York, McGraw-Hill, 1997.

45 BIGO, Didier, Pouvoir et obéissance en Centrafrique, Paris, Karthala, 1988, p. 61. 
propagandistico sulla sua persona piuttosto che su una piattaforma politica, sull'esaltazione della sua figura di "tredicesimo apostolo" 46.

Un altro fattore di consenso utilizzato in maniera assai controversa riguarda il passato coloniale e il rapporto con la Francia. Se da un lato Bokassa rivendicò costantemente le ragioni dell'indipendenza, nondimeno fin dai primi anni della sua ascesa al potere ostentò con fierezza la militanza tra le fila dell'esercito francese, e il forte legame con il suo mito, ovvero il generale De Gaulle (a cui si rivolgeva coi termini di "père" e "papa", e verso cui nutriva una vera e propria adorazione), e col presidente francese Valéry Giscard d'Estaing (che invece chiamava "mon cousin") 47. Non a caso, nel 1969, dinanzi a un parterre attonito di autorità africane, pronunziò un discorso in cui affermò: "Moi, je suis français!"48. L'ostentazione dell'appartenenza francese era funzionale alla rivendicazione di un senso di superiorità rispetto a tutti gli altri africani.

Infine, anche l'ultimo atto che testimonia della sua esasperata ricerca di legittimità e consenso, ovvero l'incoronazione a imperatore nel 1977, si inserisce in questa prospettiva contraddittoria. L'ex colonnello si autoproclamò imperatore col nome di Bokassa I, cercando, attraverso «una metamorfosi grandiosa, di riconquistare una legittimità minata da anni di dispotismo e di cattiva gestione finanziaria»49. Nei reportage e nelle foto ufficiali, il dittatore appare in abiti regali di impronta napoleonica assiso su un gigantesco trono bronzeo a forma di aquila imperiale ${ }^{50}$. Bokassa in un estremo tentativo di recuperare il consenso perduto, provò da un lato a recuperare il mito napoleonico, a testimonianza ancora una volta dello stretto legame con la Francia; dall'altro lato riprese un tema simbolico vicino alla cultura autoctona, rifacendosi all'antica tradizione degli imperi africani pre-coloniali.

Un ritorno alla tradizione è invocato anche da Mobutu quale giustificazione della sua leadership:

${ }^{46}$ Cfr. FAES, Géraldine, SMITH, Stephen, Bokassa $I_{e r}$. Un empereur français, Paris, Grasset \& Fasquelle, 2000, p. 6. Si veda anche ORIZIO, Riccardo, Parola del diavolo. Sulle tracce degli ex dittatori, Roma-Bari, Laterza, 2002.

47 Sulle relazioni tra Bokassa e il governo francese si veda BIGO, Didier, Pouvoir et obéissance en Centrafrique, op. cit., pp. 96-101.

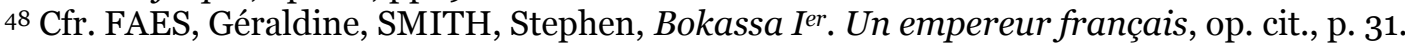

49 Ivi, p. 8.

50 HERZOG, Werner, Echi da un regno oscuro, Film sans Frontières-Sera FilmproduktionWerner Herzog Filmproduktion, Francia-Germania, 1990, 91'. Si veda anche ELKABBACH, Jean-Pierre, DUHAMEL, Alain, Cartes sur table. Sa Majesté Impériale Bokassa $1^{\text {er }}$ Empereur de Centrafrique, Antenne 2, France,1977, 57';

TITLEY, Brian, Dark Age. The Political Odyssey of Emperor Bokassa, Montreal, McGillQueen's University Press, 1997, pp. 82-104. 
doesn't it come to mind that in our African tradition, there are never two chiefs? There is sometimes a natural heir to the chief, but can anyone tell me if he has ever known an African village where there were two chiefs? This is the reason why, we Congolese, in our concern to conform to the traditions of our continent, have resolved to group together the energies of the citizens of our country under the banner of a single national party [...]. In the Congo, a chief must, and this is a necessity, seek council from the wise men. He must be informed, but after taking advice and getting information, he must make up his own mind and settle the question alone, in full knowledge of the facts ${ }^{51}$.

L'adorazione di un capo assoluto e la totale obbedienza a lui resa erano artificiosamente rappresentate come dei fattori insiti nella tradizione culturale africana, e proprio questo legame ancestrale forniva un ulteriore elemento giustificativo alla nuova deriva autoritaria del regime di Mobutu.

\section{Big Brother is watching you: poster, statue e mass-media}

Erette le fondamenta della propria venerazione, lo stadio successivo in cui appaiono impegnati i dittatori riguarda il tentativo di dare forma a una rappresentazione epifanica di tale culto. L'obiettivo era quello di procedere a un'occupazione simbolica dello spazio pubblico, e di trasformare il proprio volto e la propria effigie in una sorta di quarta dimensione della vita quotidiana, alla quale nessun cittadino avrebbe potuto sottrarsi.

Pertanto, le immagini dei dittatori campeggiavano lungo le strade delle capitali africane, impresse in manifesti ufficiali, poster celebrativi e statue. Le vie, le piazze più importanti delle città, le scuole e gli stadi erano dedicati a loro. I media e la stampa locale riportavano cronache quotidiane delle loro attività, dei loro discorsi; biografie celebrative circolavano ovunque e lo studio della loro vita divenne parte integranti dei programmi scolastici. In molti dei paesi studiati, il volto del leader era impresso perfino sulle banconote e sui francobolli. All'esterno degli edifici governativi erano esposte le loro fotografie ufficiali e quando andavano in visita ufficiale in una città, erano attesi da gruppi di ballerine che danzavano per loro, indossando spesso abiti con la loro effigie.

${ }^{51}$ MOBUTU, Sese Seko, «Address to the Conseil Nationale Extraordinaire, Dakar, 14 February 1971», in LANGLEY, Ayo J. (ed.), Ideologies of Liberation in Black Africa, 1856-1970, London, Rex Collings, 1979, pp. 722-733. 
Questo, ad esempio, accadeva nello Zaire di Mobutu, dove i funzionari dello stato erano obbligati a indossare dei risvolti che riproducevano il suo ritratto ${ }^{52}$, o in Uganda, dove i locali e gli esercizi commerciali esponevano il ritratto di Idi Amin, e i negozi di abbigliamento vendevano le magliette con la sua immagine53. Eyadéma impose l'esposizione della sua effigie in tutti gli edifici pubblici e nella capitale Lomé fu eretta una statua bronzea a lui dedicata. David Lamb, inoltre, riporta due curiosità assai indicative della diffusione e del radicamento del culto del capo nello stato togolese: secondo il giornalista americano, infatti, era possibile acquistare degli orologi da polso in cui era ritratta l'immagine del presidente, e furono persino realizzate delle tavole a fumetto dedicate a Eyadéma, in cui il dittatore assumeva le sembianze di un eroe in possesso di superpoteri ${ }^{54}$.

Di grande interesse poi i casi della Somalia e dell'Etiopia. L'attenzione sviluppata dal dittatore somalo Siad Barre verso una diffusione pervasiva della propria effigie infatti è strettamente influenzata dall'adesione all'ideologia socialista55. Le gigantografie del suo volto e i manifesti di propaganda inneggianti alla sua persona campeggiavano numerosi per le strade e sugli edifici di Mogadiscio, e rappresentavano la principale simbologia di riferimento in occasione delle cerimonie pubbliche e degli anniversari del calendario civile legato alla rivoluzione (come ad esempio la celebrazione del 21 ottobre, ricorrenza della presa del potere) ${ }^{56}$. Le imponenti parate scenografiche di militari e civili portavano in trionfo l'effigie del dittatore, e la stessa immagine si stagliava sullo sfondo dei palchi allestiti per i discorsi d'occasione. Nel caso di Barre, dunque, il culto della leadership risentiva fortemente della matrice sovietica e maoista, ed era affiancato ai simboli del marxismo, a cui il dittatore somalo provò ad attribuire una declinazione africana, integrandoli all'interno dell'immaginario collettivo autoctono.

In chiave socialista era connotato anche il culto della personalità costruito dal dittatore etiope Hailé Mariàm Menghistu ${ }^{57}$. Le statue a lui dedicate apparivano sempre inserite all'interno di un orizzonte simbolico socialista. Sintesi di questa concezione è il

\footnotetext{
${ }^{2}$ Cfr. MICHEL, Thierry, Mobutu, roi du Zaire, Les Films de la Passerelle, Belgique, 1999, 135'. 53 SCHROEDER, Barbet, Général Idi Amin Dada. Autoportrait, cit.

54 Cfr. LAMB, David, The Africans, New York, Random House, 1982, pp. 48-50.

55 Un'acuta analisi dei principali aspetti del regime di Barre si trova in COMPAGNON, Daniel, Ressources politiques, régulation autoritaire et domination personnelle en Somalie. Le régime de Siyaad Barre (1969-1991), Pau, University of Pau and Pays de l'Adour, 1995.

${ }^{6}$ Cfr. GLICKMAN, Harvey, Political Leaders of Contemporary Africa South of the Sahara, Westport, Greenwood Press, 2002.

57 Cfr. SHIFAW, Dawit, The Diary of Terror: Ethiopia 1974 to 1991, Bloomington, Trafford, 2012, pp. 32-49.
} 
memoriale della rivoluzione, monumento che rappresenta in primo piano Menghistu alla guida del popolo etiope ${ }^{5}$.

Il caso che assume anche dal punto di vista iconografico e monumentalistico i caratteri più estremi è tuttavia quello della Repubblica Centrafricana. In occasione dell'incoronazione imperiale, la capitale fu trasformata in una sorta di gigantesco tempio en plein air dedicato al culto di Bokassa, e l'intero tessuto urbano fu conquistato da un'interminabile teoria di statue, busti, poster e striscioni che ritraevano il nuovo imperatore. Nondimeno, il punto più alto della rappresentazione - sul piano visivo - di questo culto è legato alla realizzazione della corte imperiale di Berengo. A partire dal 1976, il palazzo situato nei pressi di Bobangui, città natale di Bokassa, divenne il centro del potere autocratico dell'imperatore centrafricano, sostituendo di fatto la capitale Bangui. La residenza imperiale fu assunta a luogo privilegiato dei rituali quotidiani e dei simboli (come l'aquila e il sole posti all'ingresso del complesso) legati alla persona dell'imperatore59.

L'adorazione del sovrano finì tuttavia per assumere una connotazione esasperata quando, all'inizio del 1979, Bokassa ordinò agli studenti di acquistare e indossare delle uniformi, nelle quali era stata impressa l'effigie dell'imperatore. Il nuovo provvedimento risultò eccessivamente gravoso sul piano economico e gli studenti cominciarono a manifestare. Bokassa impose la pena capitale per coloro i quali rifiutavano di indossare le uniformi e dette avvio a una brutale repressione ${ }^{60}$. Era il culmine di un vero e proprio cortocircuito semantico e simbolico che si era innescato tra le ragioni della retorica e della propaganda di regime e le reali condizioni di vita della popolazione centrafricana. Un black out che, pochi mesi dopo, contribuì a determinare il collasso del regime di Bokassa.

L'occupazione iconografica e monumentalistica dello spazio urbano fu spesso affiancata dall'occupazione dello spazio domestico e privato dei cittadini attraverso un utilizzo pervasivo dei mass media, che consentivano una efficace trasmissione del culto della personalità. Il fenomeno sociologico e culturale legato alla diffusione dei mezzi di comunicazione - in particolare quello radiofonico - è stato oggetto di numerosi studi, da cui è emerso un interessante intreccio - che andrebbe senz'altro approfondito - con l'affermarsi delle dittature militari e quindi con i meccanismi di propaganda e

${ }^{8}$ Cfr. ELLEH, Nnamdi, Architecture and Power in Africa, New York, Praeger, 2002.

59 Sul palazzo di Berengo si veda BIGO, Didier, Pouvoir et obéissance en Centrafrique, cit.; FAES, Géraldine, SMITH, Stephen, Bokassa I ${ }^{e r}$. Un empereur français, cit.; GERMAIN, Emmanuel, La Centrafrique de Bokassa, 1965-1979: force et déclin d'un pouvoir personnel, Paris, Éditions L'Harmattan, 2000.

${ }^{60}$ Cfr. DECALO, Samuel, Psychoses of Power. African Personal Dictatorships, cit. 
celebrazione della leadership ${ }^{61}$. Non a caso, in alcune circostanze, sono proprio i nuovi regimi dittatoriali a promuovere lo sviluppo di emittenti nazionali, utilizzate come strumenti di consolidamento del potere e come ulteriore risorsa nei processi di personalizzazione della politica. Del resto, come evidenzia Louise Bourgault, «the first African leaders were unusually charismatic figures, making the creation of personality cults on radio somewhat natural and self-apparent. The characteristics of the media fused with the orality of the audience and produced a solution for the political needs at hand» 62 .

Pertanto, numerosi dittatori compresero le potenzialità dei nuovi mezzi di comunicazione e, soprattutto, la possibilità di conferire alla propria leadership e al proprio culto una diffusione pervasiva e capillare. Da questo punto di vista risultano emblematiche le parole pronunciate del dittatore liberiano Samuel Kanyon Doe in un discorso diffuso dalla emittente radiofonica ufficiale del regime liberiano:

\begin{abstract}
For too long the masses of the Liberian people have been kept in the dark about how their own country is being run. For too long, the vast majority of the Liberian people have been prevented from participating in the making of decisions that affect their daily lives. One of the principal reasons why our people have been kept in the dark and have not participated in the running of Liberia is the absence of a mass communications system for the entire country. In order for any people anywhere to feel that they are an important part of a country and be able to promote development, they must have an effective means for making their voices heard and they must receive regular reports on how the country is run [...]. It is for these reasons that the People's Redemption Council Government has made all efforts to take rapid action in facilitating the setting up of a broadcasting system that will serve not just a handful of people in Monrovia, but the masses of the people throughout Liberia. Such a broadcasting system will keep the people informed on a regular basis about how the country is being run and will motivate
\end{abstract}

\footnotetext{
${ }^{61}$ Cfr. FARDON, Richard, FURNISS, Graham (eds.), African Broadcast Cultures: Radio in Transition, Oxford, James Currey, 2001; LENOBLE-BART, Annie, TUDESQ, André-Jean (sous la direction de), Connaître les médias d'Afriques subsaharienne. Problématiques, sources et ressources, Paris, Karthala, 2008; TUDESQ, André-Jean, La radio en Afrique noire, Paris, Pedone, 1983; ID., L'Afrique noire et ses télévisions, Paris, Anthropos, 1992; ID., L'Afrique parle, l'Afrique écoute. Les radios en Afrique subsaharienne, Paris, Karthala, 2002. 62 BOURGAUlT, Louise M., Mass Media in Sub-Saharan Africa, Bloomington, Indiana University Press, 1995, p. 77.
} 
them to make suggestions to the government. It will encourage the people to work very hard so as to able to be able to enjoy the fruits of their labor63.

La ricerca di un'adesione partecipativa del popolo al programma politico del regime costituiva un efficace pretesto alla martellante propaganda perseguita attraverso le reti radiofoniche e televisive.

I bollettini ufficiali trasmessi nei vari paesi attraverso i nuovi mass media presentavano giornalmente le notizie della vita pubblica dei dittatori, delle loro attività e mandavano in onda i loro discorsi alla popolazione. Perfino, nelle sale cinematografiche all'inizio di ogni proiezione erano mostrati dei video di propaganda che li ritraevano nelle gesta eroiche o nel servizio prestato alla patria. Ad esempio, nello stato zairese, all'inizio di ogni programma televisivo, le emittenti di stato mostravano la figura di Mobutu nell'atto di venir fuori dalle nuvole e discendere verso la Terra, in una sorta di icona della trasfigurazione ${ }^{64}$.

Anche Bokassa si servì del mezzo radiofonico, in primo luogo come strumento di diffusione dei suoi slogan politici, in particolare quelli che lo rappresentavano come il padre dei centrafricani: "le militaire que je suis est aussi un bon papa", "les enfants doivent tout dire à leur père, ils n'ont rien à lui cacher" ${ }^{65}$. Nondimeno, fu in occasione della sua incoronazione a imperatore - attraverso la promozione di un'intensa campagna propagandistica - che Bokassa sfruttò in pieno le potenzialità della comunicazione radiofonica ${ }^{66}$.

L’importanza dei nuovi media era tale che in alcuni casi i capi militari assumevano personalmente la direzione della radio di stato, o comunque seguivano in maniera diretta l'organizzazione dei programmi e la gestione della propaganda ${ }^{67}$.

\section{Conclusioni}

Considerati dal mondo occidentale - a seconda delle immagini trasmesse dalla stampa e dai media ${ }^{68}$ - come dei personaggi grotteschi e megalomani, dei leader

63 GIVENS, Willie A. (ed.), Liberia: The Road to Democracy Under the Leadership of Samuel Kanyon Doe. The Policies and Public Statements of Dr Samuel Kanyon Doe, Bucks, Kensal Press, 1986, pp. 43-44.

64 Cfr. RUBIN, Barry, Modern Dictators, cit., pp. 94-95.

65 BIGO, Didier, Pouvoir et obéissance en Centrafrique, cit., p. 64.

${ }^{66}$ Cfr. CONDOM, Cédric, Notre ami l'empereur Bokassa I $I_{e r}$, Kilaohm Productions, France, 2011, $52^{\prime}$.

${ }_{67}$ Cfr. TUDESQ, André-Jean, L'Afrique parle, l'Afrique écoute. Les radios en Afrique subsaharienne, cit., 1983, p. 105. 
spietati e sanguinari, degli psicopatici, dei buffoni o degli showman, in realtà i capi delle dittature militari hanno mostrato una precisa strategia politica, fondata anche sull'esaltazione della propria leadership. Il culto della personalità che questi satrapi moderni hanno costruito rappresenta uno degli strumenti attraverso cui hanno provato a conferire legittimità al regime imposto con le armi.

L'opinione pubblica europea e, spesso, perfino i governi hanno guardato a questa particolare fenomenologia politica con lo sguardo della curiosità o dell'indignazione, senza tuttavia indagare a fondo le ragioni di un contesto così lontano dalla propria prospettiva. Come sottolinea ad esempio Didier Bigo in riferimento al caso centrafricano - ma la stessa considerazione può essere estesa anche alle altre dittature del continente -, la «croyance solidement enracinée de trouver une réponse [...] en assimilant Bokassa à un malade mental, occulte la dimension politique du problème. Le couronnement dans les oripeaux et les défroques napoléoniennes, ne peut être ramené à un délire mégalomaniaque où Bokassa n'est rien d'autre qu'un des empereurs de pacotille qui hantent les asiles d'aliénés» ${ }^{69}$.

Proprio questa barriera pregiudiziale ha rappresentato il limite principale nello studio scientifico di tali aspetti. In realtà l'analisi del culto della personalità edificato da questi dittatori rivela una nuova dimensione della politica e del potere, mettendo in discussione i riferimenti e i criteri di classificazione tradizionali dei sistemi occidentali. In particolare, attraverso lo studio di tali dinamiche è possibile fare luce su alcuni interrogativi cruciali: «comment le pouvoir est-il ressenti? Comment ses excés sont-ils reçus et interprétés par la population? Quel est le rapport entre le pouvoir et l'obéissance?»70. Del resto, la riflessione sul ruolo della leadership rimanda alla più ampia questione della gestione personale delle istituzioni statali nell'Africa postcoloniale e, inevitabilmente, alla categoria del neopatrimonialismo, che, proprio in questi contesti, trova numerosi e concreti riscontri ${ }^{71}$.

68 Cfr. SANCHEZ PIÑOL, Albert, Pagliacci e mostri. Storia tragicomica di otto dittatori africani, Milano, Libri Scheiwiller, 2009.

69 BIGO, Didier, «Approche pour une théorie du pouvoir personnel, un exemple privilégié: le Centrafrique», in Etudes polémologiques, 36, 4/1985, p. 68. Emblematiche, sotto questo profilo, anche le frasi con cui Idi Amin fu definito da due importanti riviste occidentali, ovvero il Sunday Mirror e il Time magazione, che scrissero rispettivamente "He's nuts" e "The Wild Man of Africa". Cfr. WIEDEMANN, Erich, Idi Amin un eroe dell'Africa?, Milano, Sonzogno Dossier, 1977, p. 248.

70 GERMAIN, Emmanuel, La Centrafrique de Bokassa, 1965-1979: force et déclin d'un pouvoir personnel, op. cit., p. 15 .

${ }^{71} \mathrm{Su}$ questi temi si vedano i numerosi studi di Jéan-François Médard, e in particolare, «L'État patrimonialisé», in Politique africaine 39, 1990, pp. 25-36; «L'État néo-patrimoniale en Afrique Noire», in MÉDARD, Jéan-François, États d'Afrique noire. Formation, mécanismes et crise, Paris, Karthala, 1991, pp. 323-353. 
Emergono dunque delle implicazioni nuove che costringono a rivisitare il ruolo dei miti, dei simboli e della coscienza collettiva nell'agone politico e forniscono allo stesso tempo nuove chiavi interpretative per le dinamiche politiche dell'occidente moderno ${ }^{72}$.

${ }^{2}$ Cfr. BACH, Daniel, GAZIBO, Mamoudou (eds.), Neopatrimonialism in Africa and Beyond, London, Routledge, 2012. 


\section{${ }^{*}$ L'autore}

Claudio Mancuso è dottore di ricerca in Storia dei partiti e dei movimenti politici (Università degli Studi di Urbino). Si occupa dello studio dei processi di rappresentazione simbolica del potere politico e dei meccanismi di costruzione del consenso in epoca moderna e contemporanea. In particolare, nelle sue ultime ricerche, ha affrontato la questione legata all'evoluzione dei sistemi di propaganda durante la Prima guerra mondiale e negli anni della Guerra fredda.

URL: < http://www.studistorici.com/progett/autori/\#Mancuso >

\section{Per citare questo articolo:}

MANCUSO, Claudio, "Corone, guerrieri e redentori. II culto della personalità nelle dittature militari dell'Africa subsahariana negli anni dellla Guerra Fredda», Diacronie. Studi di Storia Contemporanea : Le dittature militari: fisionomia ed eredità politica, 29/12/2015,

URL:< http://www.studistorici.com/2015/12/29/mancuso_numero_24/ >

Diacronie Studi di Storia Contemporanea $\widehat{\vartheta}$ www.diacronie.it

Risorsa digitale indipendente a carattere storiografico. Uscita trimestrale. redazione.diacronie@hotmail.it

Comitato di redazione: Jacopo Bassi - Luca Bufarale - Elisa Grandi - Antonio César Moreno Cantano - Deborah Paci - Fausto Pietrancosta - Alessandro Salvador - Matteo Tomasoni - Luca Zuccolo

Diritti: gli articoli di Diacronie. Studi di Storia Contemporanea sono pubblicati sotto licenza Creative Commons 3.0. Possono essere riprodotti e modificati a patto di indicare eventuali modifiche dei contenuti, di riconoscere la paternità dell'opera e di condividerla allo stesso modo. La citazione di estratti è comunque sempre autorizzata, nei limiti previsti dalla legge. 